\title{
Effect of non-oil-seed pulses on glycaemic control: a systematic review and meta-analysis of randomised controlled experimental trials in people with and without diabetes
}

\author{
J. L. Sievenpiper • C. W. C. Kendall • A. Esfahani • \\ J. M. W. Wong • A. J. Carleton • H. Y. Jiang • \\ R. P. Bazinet • E. Vidgen • D. J. A. Jenkins
}

Received: 29 January 2009 / Accepted: 30 March 2009/Published online: 13 June 2009

(C) Springer-Verlag 2009

\begin{abstract}
Aims/hypothesis Dietary non-oil-seed pulses (chickpeas, beans, peas, lentils, etc.) are a good source of slowly digestible carbohydrate, fibre and vegetable protein and a valuable means of lowering the glycaemic-index (GI) of the diet. To assess the evidence that dietary pulses may benefit glycaemic control, we conducted a systematic review and meta-analysis of randomised controlled experimental trials investigating the effect of pulses, alone or as part of low-GI or high-fibre diets, on markers of glycaemic control in people with and without diabetes.

Methods We searched MEDLINE, EMBASE, CINAHL, and the Cochrane Library for relevant controlled trials of
\end{abstract}

A. Esfahani and J. M. W. Wong contributed equally to this study.

Electronic supplementary material The online version of this article (doi:10.1007/s00125-009-1395-7) contains supplementary material, which is available to authorised users.

\footnotetext{
J. L. Sievenpiper $\cdot$ C. W. C. Kendall $\cdot$ A. Esfahani $\cdot$

J. M. W. Wong • A. J. Carleton · H. Y. Jiang $\cdot$ E. Vidgen •

D. J. A. Jenkins

Risk Factor Modification Centre, St Michael's Hospital,

Toronto, ON, Canada
}

\section{J. A. Jenkins}

Division of Endocrinology, St Michael's Hospital,

Toronto, ON, Canada

J. L. Sievenpiper $\cdot$ C. W. C. Kendall $(\bowtie) \cdot$ A. Esfahani $\cdot$

J. M. W. Wong • A. J. Carleton $\cdot$ H. Y. Jiang $\cdot$ R. P. Bazinet

E. Vidgen • D. J. A. Jenkins

Department of Nutritional Sciences, Faculty of Medicine,

University of Toronto,

The FitzGerald Building, Room 340, 150 College Street,

Toronto, ON M5S 3E2, Canada

e-mail: cyril.kendall@utoronto.ca $\geq 7$ days. Two independent reviewers (A. Esfahani and J. M. W. Wong) extracted information on study design, participants, treatments and outcomes. Data were pooled using the generic inverse variance method and expressed as standardised mean differences (SMD) with 95\% CIs. Heterogeneity was assessed by $\chi^{2}$ and quantified by $I^{2}$. Meta-regression models identified independent predictors of effects.

Results A total of 41 trials (39 reports) were included. Pulses alone (11 trials) lowered fasting blood glucose (FBG) $(-0.82,95 \% \mathrm{CI}-1.36$ to -0.27$)$ and insulin $(-0.49,95 \%$ $\mathrm{CI}-0.93$ to -0.04$)$. Pulses in low-GI diets (19 trials) lowered glycosylated blood proteins (GP), measured as $\mathrm{HbA}_{1 \mathrm{c}}$ or

D. J. A. Jenkins

Department of Medicine,

Faculty of Medicine,

University of Toronto,

Toronto, ON, Canada

\section{W. C. Kendall}

College of Pharmacy and Nutrition,

University of Saskatchewan,

Saskatoon, SK, Canada

H. Y. Jiang

Michael G DeGroote School of Medicine,

McMaster University,

Hamilton, ON, Canada 
fructosamine $(-0.28,95 \% \mathrm{CI}-0.42$ to -0.14$)$. Finally, pulses in high-fibre diets (11 trials) lowered FBG $(-0.32,95 \% \mathrm{CI}$ -0.49 to -0.15$)$ and GP $(-0.27,95 \%$ CI -0.45 to -0.09$)$. Inter-study heterogeneity was high and unexplained for most outcomes, with benefits modified or predicted by diabetes status, pulse type, dose, physical form, duration of followup, study quality, macronutrient profile of background diets, feeding control and design.

Conclusions/interpretation Pooled analyses demonstrated that pulses, alone or in low-GI or high-fibre diets, improve markers of longer term glycaemic control in humans, with the extent of the improvements subject to significant interstudy heterogeneity. There is a need for further large, welldesigned trials.

Keywords Beans · Chickpeas · Diabetes · Glycaemia Lentils $\cdot$ Peas $\cdot$ Pulses

$\begin{array}{ll}\text { Abbreviations } \\ \% \text { PE } & \text { Per cent of energy } \\ \text { ADA } & \text { American Diabetes Association } \\ \text { GI } & \text { Glycaemic index } \\ \text { GP } & \text { Glycosylated blood proteins } \\ \text { FBG } & \text { Fasting blood glucose } \\ \text { FBI } & \text { Fasting blood insulin } \\ \text { HOMA-IR } & \text { HOMA of insulin resistance } \\ \text { IGT } & \text { Impaired glucose tolerance } \\ \text { MQS } & \text { Methodological quality score } \\ \text { SMD } & \text { Standardised mean difference }\end{array}$

\section{Introduction}

Diabetes has become one of the most important unmet prevention and treatment challenges. Despite the growing armamentarium of medications, which include five new classes since metformin was first approved in 1995 in the USA [1, 2], the combined prevalence of impaired glucose tolerance (IGT) and diabetes has surpassed 13\% worldwide and continues to grow [3]. Although oral antihyperglycaemic agents have been shown to prevent the development of diabetes in high-risk individuals and to reduce the risk of microvascular complications in individuals with type 2 diabetes, they have failed to deliver the anticipated macrovascular benefits [4-6]. There are, however, a few exceptions $[7,8]$, such as the $\alpha$-glucosidase inhibitor acarbose, which has been shown to decrease incident diabetes in individuals with IGT [9] and cardiovascular events in individuals with type 2 diabetes [8].

Various dietary interventions share similarities with acarbose, which slows the rate of absorption such that dietary carbohydrate is converted into slowly absorbed or 'lente' carbohydrate, reducing postprandial glucose and insulin excursions and effectively lowering the glycaemic index (GI) of the diet. Dietary pulses (dried leguminous seeds, including chickpeas, beans, peas and lentils) are a good source of amylose starch, fibre, vegetable protein and antinutrients, such as phytates, phenols, lectins and enzyme inhibitors, some of which may act as natural inhibitors of both $\alpha$-amylase and $\alpha$-glucosidase [10]. In early studies, pulses were shown to result in exceptionally low glycaemic responses when fed to healthy volunteers [11], and in later experiments were demonstrated to possess a carbohydrate component that was more slowly digested than that of other foods such as cereals [12]. This property of slower absorption, which is common to $\alpha$-glucosidase inhibitors, makes pulses an important means of lowering the GI of the diet, a characteristic that has been exploited extensively in studies of low-GI diets [13-31], and would therefore be predicted to benefit glycaemic control and, by analogy with acarbose, CHD risk [8]. Based on limited clinical experimental evidence, the European (EASD) [32], Canadian (CDA) [33], and American (ADA) [34] diabetes associations recommend the consumption of dietary pulses as a means of optimising diabetes control through lowering the GI and increasing the dietary fibre content of the diet. To address the need for better clinical evidence to support nutrition recommendations for diabetes, we conducted a systematic review and meta-analysis of randomised clinical experimental trials investigating the effect of non-oil-seed dietary pulses, alone or as part of a low-GI or high-fibre diet, on markers of glycaemic control in people with and without diabetes.

\section{Methods}

We followed the Cochrane Handbook for Systematic Reviews of Interventions Version 5.0.0, updated February 2008, for the planning and conduct of this meta-analysis [35]. The reporting followed the Quality of Reporting of Meta-analyses (QUOROM) guidelines [36].

Study selection We conducted a search of Medline (1950-), EMBASE (1980-), CINAHL (1982-) and the Cochrane Library (including The Cochrane Central Register of Controlled Trials [Clinical Trials; CENTRAL] database [1800-]) up to 22 May 2008, using the following search terms and Boolean operators: 'beans OR bean OR lentils OR lentil OR chickpeas OR chickpea OR peas OR pea OR legume OR legumes OR leguminous OR lupin OR lupins OR vetch OR golden gram OR black gram OR green gram OR Bambara groundnut OR bambarra groundnut AND (glucose OR OGTT OR $\mathrm{HbA}_{1 \mathrm{c}}$ OR $\mathrm{A}_{1 \mathrm{c}}$ OR fructosamine OR insulin OR diabetes)'. The terms bean(s) (red and white 
kidney, black, pinto, fava, and white [white, navy, haricot], black, brown, long, and mung, etc.) and pea(s) (yellow, green, split, black-eyed, cow, sugar, snap, snow, etc.) were used to capture specific pulses that have these words in their names. The search was restricted to human research studies. No limit was placed on language. Manual searches supplemented the database search strategy. We included randomised controlled experimental trials that investigated the medium- to long-term effect of supplementing the diet with non-oil-seed pulses in food form, alone or as part of a low-GI or a high-fibre dietary intervention, compared with a non-pulse control intervention on indices of glycaemic control in humans. Studies that were not randomised, had a follow-up period of $<7$ days, contained oil-seed pulses (peanuts or soy beans) or non-oil-seed pulses in extract or tablet/capsule form, lacked a suitable control intervention, or reported unbalanced or hyperenergetic comparisons, were excluded. We also excluded studies that contained multiple, concurrent interventions on the pulses' treatment arm, except studies in which low-GI or high-fibre dietary interventions included pulses. In cases where multiple publications with duplicate data existed for the same study, the article with the most information was included.

Data extraction Two investigators (J. M. W. Wong, A. Esfahani) independently extracted relevant data on study characteristics and outcomes using a standardised proforma. These data included information on study design (parallel, crossover, factorial, etc.); randomisation; blinding; sample size; participant characteristics (age, sex, BMI, diabetes status and presence of preexisting conditions [diabetes, metabolic syndrome, hypercholesterolaemia, coronary artery disease, CHD]); pulse type, form and dose; control diets; duration of follow-up and macronutrient profile of the background diet. Mean \pm SEM values for fasting (glucose, insulin, $\mathrm{HbA}_{1 \mathrm{c}}$ and fructosamine concentrations and insulin sensitivity as assessed by HOMA of insulin resistance [HOMA-IR]) or postprandial (glucose and insulin concentrations and derived means, AUC and insulin sensitivity) variables were extracted. Studies that did not report SEM values had these values imputed from the SD, 95\% CIs, $p$ values, $T$ and $F$ statistics, using standard formulae [35]. If these data were unavailable, then SEM was calculated from an SD that was extrapolated by imputing the pooled SD from the other studies included in the meta-analysis, according to Furukawa et al. for unpaired data [37] and Elbourne et al. [38] for paired data. Studies that differed in the units used to report concentrations had their units converted using standard conversion factors. Where HOMA-IR was not reported, it was imputed using the formula of Matthews et al. [39]. Missing SEMs for HOMA-IR were imputed according to the standard formulae for functions of two variables [40]. The investigators also assessed the quality of each study using the Heyland score [41], which assigns a score from 0 to 1 or 0 to 2 over nine categories of quality related to study design (randomisation, blinding, protocol analysis), sampling procedures (selection, compatibility, follow-up), and interventions (protocol description, co-interventions, crossovers) to give a total of up to 13 points (Electronic supplementary material [ESM] Table 1). Studies that reported $100 \%$ follow-up data were scored as intention-to-treat analyses. Designs with metabolic feeding control, where participants were provided with all foods on the study diets and all unused foods were returned for assessment of compliance, were also recorded as a measure of study quality. Disagreements were reconciled by consensus and, where necessary, by discussion with J. L. Sievenpiper or C. W. C. Kendall. Authors were not contacted to request additional information, except for one author who was contacted regarding the use of pulses in their study [23].

Statistical analyses Data were analysed using Review Manager (RevMan) 5.0.17 (Cochrane Library software, Oxford, UK). Three separate pooled analyses were conducted using the generic inverse variance method with inverse variance weighting for pulses alone, pulses in lowGI dietary interventions and pulses in high-fibre dietary interventions. Outcomes included glycosylated blood proteins (GP; $\mathrm{HbA}_{1 \mathrm{c}}$ or fructosamine, with $\mathrm{HbA}_{1 \mathrm{c}}$ preferred if the duration of the trial was longer than 12 weeks), fasting blood glucose (FBG) and insulin (FBI) concentrations and HOMA-IR, with change from baseline differences preferred to differences at study end between pulse and control interventions. Paired analyses were applied to all crossover trials, according to Elbourne et al. [38], necessitating the expression of data as standardised mean differences (SMD) with 95\% CIs, where SMDs are interpreted as follows: $<0.4$, small effect size; $0.4-0.7$, moderate effect size; and $>0.7$, large effect size. To address the possible introduction of a unit-of-analysis error by including trials with multiple intervention arms compared with a single control arm, we combined pulse-containing arms to create a single pair-wise comparison. Inter-study heterogeneity was tested by Cochrane's $Q\left(\chi^{2}\right.$ at a significance level of $\left.p<0.10\right)$ and quantified by $I^{2}$, where $I^{2} \geq 50 \%$ is considered to be evidence of substantial heterogeneity and $\geq 75 \%$, considerable heterogeneity [35]. Random effects models were applied for instances where heterogeneity was significant; fixed effects models were used otherwise. Sources of heterogeneity were investigated by sensitivity analyses, in which the effect of systematically removing each study was assessed. A priori subgroup analyses were also undertaken to investigate sources of heterogeneity, including the effect of participant category (normoglycaemia, type 1 diabetes, type 2 diabetes, any diabetes); pulse type (Phaseolus vulgaris [black, white, pinto, red and white kidney beans], 
Cicer arietinum [chickpeas], Vicia faba [fava beans], Vigna unguiculata [black-eyed peas]), dosage $(<$ and $\geq 100 \mathrm{~g} /$ day) [42] and physical form (whole, flour, fibre); duration of follow-up ( $\leq$ and $>4$ weeks) and study quality according to the Heyland methodological quality score (MQS; $<$ and $\geq 8$ ) [41] on both the pooled effect estimates and their heterogeneity. Additional post hoc subgroup analyses were undertaken to investigate the effect of the macronutrient profile (carbohydrate $<$ and $\geq 55 \%$ of energy [\%E], fat $\leq$ and $>30 \% \mathrm{E}$, protein $<$ and $\geq 20 \% \mathrm{E}$ ) [32-34], and fibre content ( $<$ and $\geq 40$ g/day) [32-34] of the background diet; GI ( $\leq$ and $>70$ [bread scale]) of the test diet and difference in GI between the test and control diets ( $\Delta$ GI $\leq$ and $>15$ ) [43, 44]; level of feeding control (metabolic, non-metabolic), study design (parallel, crossover), and use of washout periods (yes, no) in crossover trials. Adjustment for multiple comparisons was made in the post hoc subgroup analyses using the Bonferroni procedure. Multivariate analyses were performed by meta-regression, where inverse variance-weighted multiple regression models were selected by All Possible Regression using the adjusted $R^{2}$ criterion (Number Cruncher Statistical System software; NCSS, Kaysville, UT, USA). Publication bias was investigated using Funnel plots.

Role of the sponsor The sponsors had no role in the design, conduct, or reporting of the present study.

\section{Results}

Search results Figure 1 shows the flow of the literature when the systematic search and selection strategies were applied. The initial search identified 1,014 reports, of which 749 were excluded based on the title or abstract, leaving 265 reports eligible for full review. Of these reports, 226 were excluded based on other criteria, leaving 41 trials (39 reports) that satisfied the inclusion criteria but did not meet the exclusion criteria, of which 11 trials (ten reports) investigated pulses alone, 19 trials (19 reports) investigated pulses in a low-GI dietary intervention, and 11 trials (ten reports) investigated pulses in a high-fibre dietary intervention.

Trial characteristics Tables 1,2, and 3 show the characteristics of the 41 randomised controlled experimental trials included in each of the three meta-analyses: pulses alone [45-54], pulses in low-GI diets [13-31] and pulses in highfibre diets [55-64]. The 11 trials (ten reports) investigating pulses alone (Table 1) were predominantly crossover in design (seven trials, five of which had a washout period), studied a total of 253 participants (type 2 diabetes, $n=21$; normoglycaemia and/or hypercholesterolaemia, $n=232$ ) and had a mean sample size of 23. Pulse types included chickpeas, black-eyed peas and various beans (red and white kidney, black, pinto, fava and white [white, navy and haricot]), and the mean dosage was $152.1 \mathrm{~g} /$ day (range 15.5-465 g/day). The mean duration of study follow-up was 6.7 weeks (range 1-96 weeks). Background diets were largely high-carbohydrate, low-fat diets, consisting of $52 \% \mathrm{E}$ carbohydrate (range $43-62 \% \mathrm{E}$ ), $18 \% \mathrm{E}$ protein (range $15-20 \% \mathrm{E}$ ) and $29 \% \mathrm{E}$ fat (range $20-37 \% \mathrm{E}$ ). Study quality assessed by the Heyland MQS ranged from 4-9, with only four of the 11 trials considered to be of high quality (MQS $\geq 8$ ). Only three trials were metabolically controlled.

The 19 trials investigating pulses in low-GI diets (Table 2) were predominantly crossover in design (12 trials, ten of which had a washout period), studied a total of 762 participants (type 2 diabetes, $n=420$; type 1 diabetes, $n=130$; normoglycaemia, $n=212$ ) and had a mean sample size of 40 . Pulse types included beans (white [white, navy and haricot], pinto, kidney, black, brown, long and mung), split peas, chickpeas, lentils and unspecified legumes; dosing information was not available. The mean GI of the pulse-containing low-GI diets was 66.9 (range 54.3-79). The mean duration of study follow-up was 14.3 weeks (range 2-52 weeks). Background diets were largely high-carbohydrate, low-fat diets, consisting of $50 \% \mathrm{E}$ carbohydrate (range $36-60 \% \mathrm{E}$ ), $20 \% \mathrm{E}$ protein (range $12-26 \% \mathrm{E}$ ) and $29 \% \mathrm{E}$ fat (range $21-$ $42 \% \mathrm{E}$ ). The mean fibre content of the pulse-containing lowGI diets was $33 \mathrm{~g} /$ day (range 12-72 g/day). Study quality assessed by the Heyland MQS ranged from 5 to 8, with ten of 19 trials considered to be of high quality (MQS $\geq 8$ ). Only four trials were metabolically controlled.

The 11 trials (ten reports) investigating pulses in highfibre diets (Table 3) were predominantly crossover in design (nine trials, only three of which had a washout period) and studied a total of 641 participants (type 2 diabetes, $n=140$; type 1 diabetes, $n=89$; normoglycaemia, $n=412$ ), with a mean sample size of 58 . Pulse types included lima beans; green and yellow peas; green, white (white, navy, haricot), brown and pinto beans; chickpeas; lentils; unspecified legumes; and lupin bean kernel fibre; dosing information was not available. The mean fibre content of the pulse-containing high-fibre diets was $58 \mathrm{~g}$ /day (range 26.9-96.6 g/day). The mean duration of the follow-up period was 19.9 weeks (range 1.4-156 weeks). Background diets were largely high-carbohydrate, low-fat diets, consisting of a mean of $51.9 \% \mathrm{E}$ carbohydrate (range $23-70 \% \mathrm{E}$ ), $19.6 \% \mathrm{E}$ protein (range $13-62 \% \mathrm{E}$ ) and $29.8 \% \mathrm{E}$ fat (range $10-55 \% \mathrm{E}$ ). Study quality assessed by the Heyland MQS ranged from 3 to 9 , with six of 11 trials considered to be of high quality (MQS $\geq 8$ ). Only three trials were metabolically controlled. 
Fig. 1 Flow of the literature

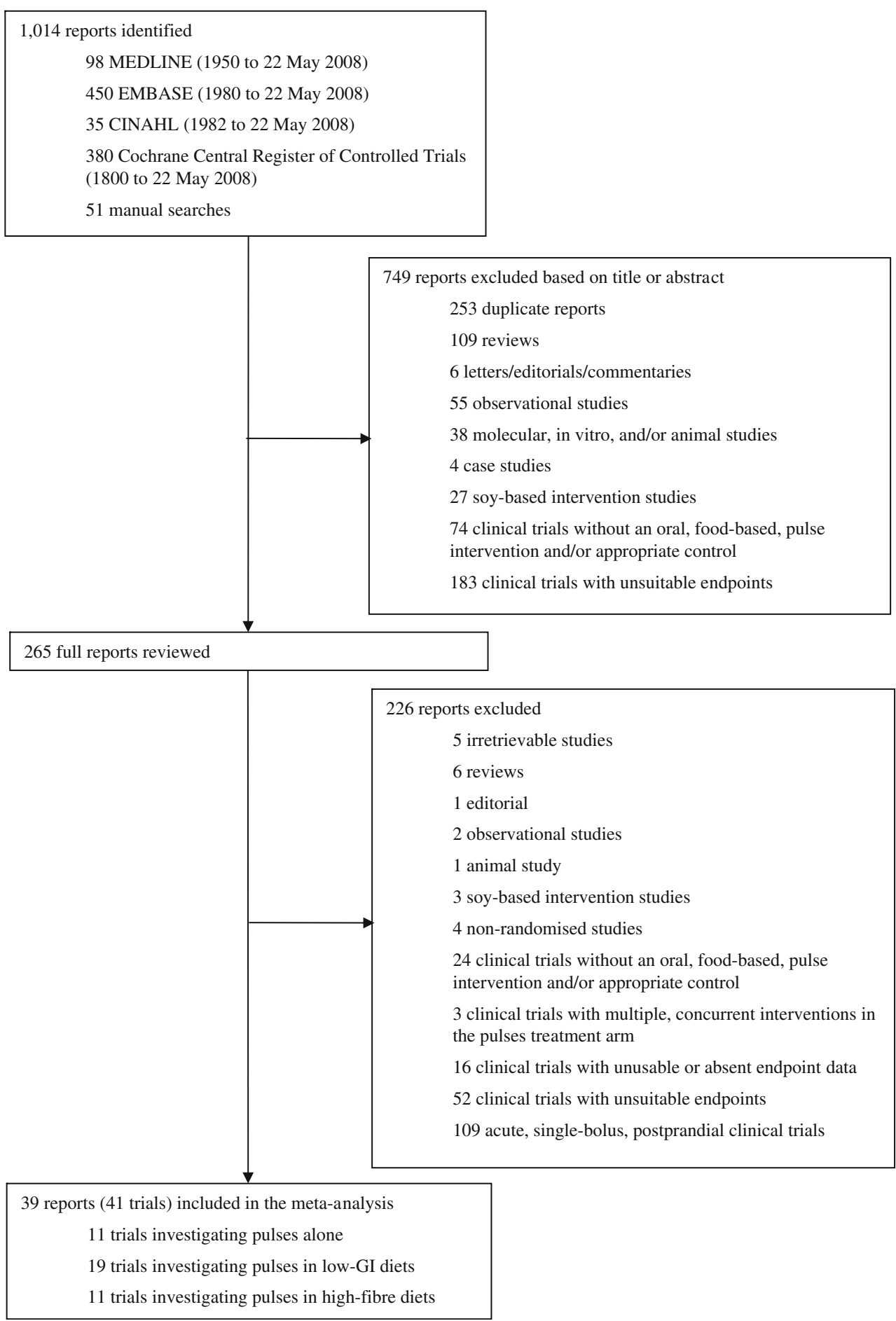

Pulses alone Figure 2 shows forest plots for the effect of pulses alone on FBG, FBI, HOMA-IR and GP. Pooled analyses showed that FBG $(-0.82,95 \% \mathrm{CI}-1.36$ to -0.27$)$ and FBI $(-0.49,95 \% \mathrm{CI}-0.93$ to -0.04$)$ were significantly decreased by pulses alone, whereas HOMA-IR and GP were not. There was evidence of considerable $\left(I^{2} \geq 75 \%\right)$, significant $(p<0.10)$ inter-study heterogeneity for all out- comes. Systematic removal of each trial during sensitivity analyses did not alter the significance of the effect estimate or heterogeneity for any of the outcomes, with one exception. Removal of Fruhbeck et al. [49] from the analysis eliminated the evidence for significant inter-study heterogeneity for FBI $\left(I^{2}=0 \%, p=0.47\right)$ without altering the significant benefit of pulses alone on this outcome. 


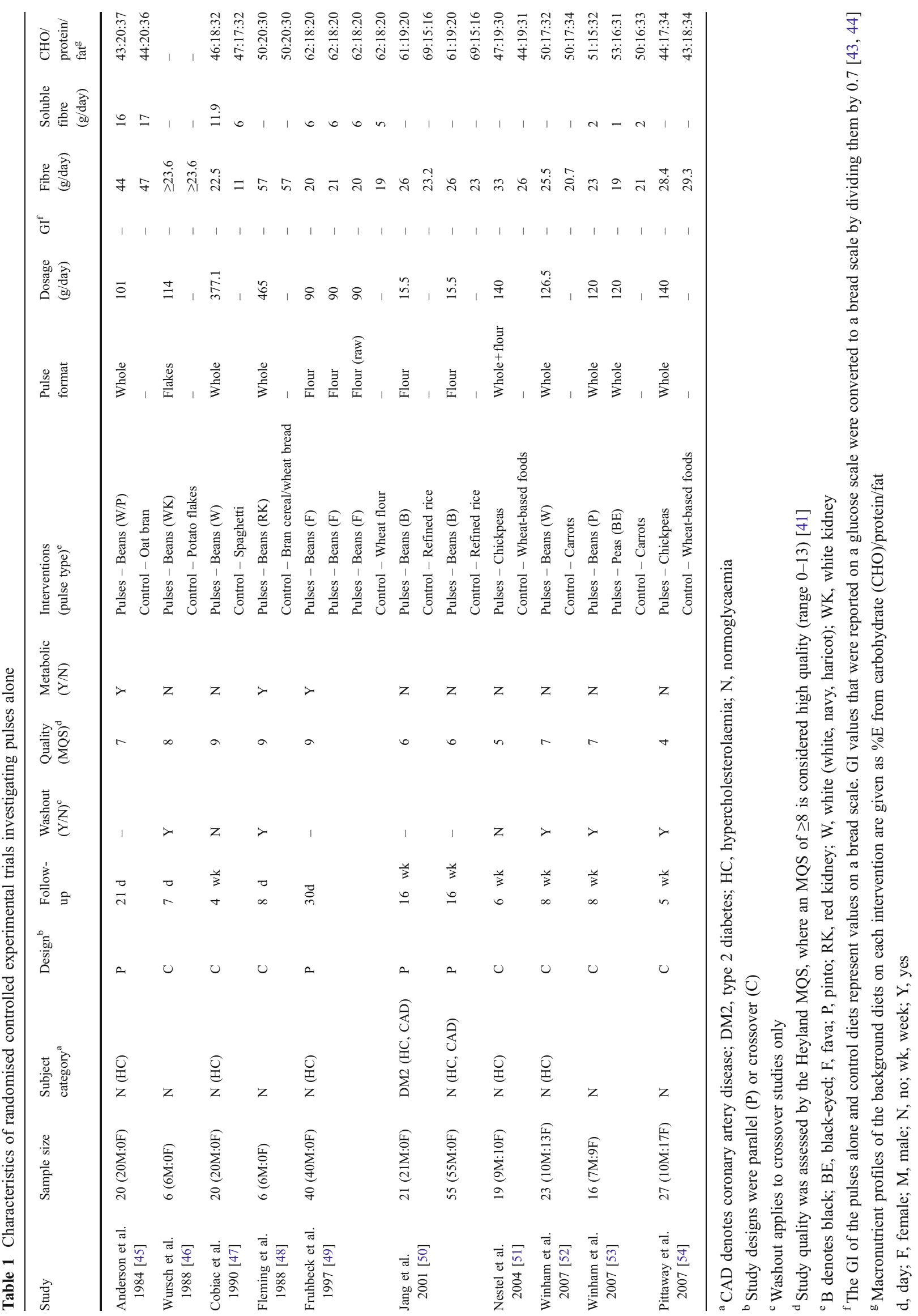


Pulses in low-GI diets Figure 3 shows forest plots for the effect of pulses in low-GI diets on FBG, insulin, HOMA-IR and GP. Pooled analyses showed that the level of GP was significantly decreased $(-0.28,95 \%$ CI -0.42 to -0.14$)$, whereas FBG, FBI and HOMA-IR were not. There was evidence of substantial $\left(I^{2} \geq 50 \%\right)$, significant $(p<0.10)$ inter-study heterogeneity for all outcomes, with the exception of FBI $\left(I^{2}=0 \%, p=0.47\right)$. Systematic removal of each trial during sensitivity analyses did not alter the significance of the effect estimate or heterogeneity for any of the outcomes, with one exception. Removal of Bouche et al. [23] eliminated the evidence for significant inter-study heterogeneity for HOMA-IR $\left(\mathrm{I}^{2}=20 \%, p=0.29\right)$ without altering the significant lack of effect of pulses in low-GI diets on this outcome.

Pulses in high-fibre diets Figure 4 shows forest plots for the effect of pulses in high-fibre diets on FBG, FBI, HOMA-IR and GP. Pooled analyses showed that FBG $(-0.32,95 \%$ CI -0.49 to -0.15$)$ and GP $(-0.27,95 \%$ CI -0.45 to -0.09 ) were significantly decreased by pulses in high-fibre diets, whereas FBI and HOMA-IR were not. There was evidence of considerable $\left(I^{2} \geq 75 \%\right)$, significant $(p<0.10)$ inter-study heterogeneity for FBG and GP only. Systematic removal of each trial during sensitivity analyses did not alter the significance of the effect estimate or heterogeneity for any of the outcomes.

Univariate subgroup analyses Univariate a priori and post hoc subgroup analyses explored the effect of sources of heterogeneity on the effect of pulses, alone or as part of low-GI or high-fibre dietary interventions, on FBG, FBI, HOMA-IR and GP (ESM Tables 2, 3, and 4). Inter-study heterogeneity for a benefit or lack of benefit of pulses was explained by only a few subgroup analyses, as defined by a loss of significance by Cochrane's $Q\left(\chi^{2}\right)$ in one or more of the subgroup categories. Otherwise, effects were found to be dependent on various subgroup categories, with persistent substantial to high heterogeneity $\left(p<0.10, I^{2} \geq 50 \%\right)$ observed across the three pooled analyses. The most robust modifiers of benefit in a priori subgroup analyses across the three pooled analyses were type 2 diabetes (two of four outcomes in the analysis of pulses in low-GI diets, and two of two outcomes in the analysis of pulses in high-fibre diets), follow-up $>4$ weeks (two of three outcomes in the analysis of pulses alone, and one of two outcomes in the analysis of pulses in high-fibre diets), and MQS $<8$ (two of three outcomes in the analysis of pulses alone, and one of four outcomes in the analysis of pulses in low-GI diets). Specific to the pulses alone analysis, pulse species including Phaseolus vulgaris (black, white, pinto, red and white kidney beans), Cicer arietinum (chickpeas) and Vicia faba (fava beans) were also identified as significant modifiers. In post hoc subgroup analyses, the most robust modifier of benefit across the three pooled analyses after adjustment by the Bonferroni procedure was metabolic feeding control (GP in the analysis of pulses in low-GI diets, and FBG and GP in the analysis of pulses in highfibre diets). Otherwise, significant modifiers tended to be specific to each analysis. In the analysis of pulses in low-GI diets, the pulses-mediated decrease in GP was dependent on a GI of $\leq 70$ on the low-GI diet and a difference in GI between interventions ( $\Delta \mathrm{GI}$ ) of $>15$. Similarly, in the highfibre pooled analysis, the pulses-mediated decrease in FBG was dependent on a fibre intake of $\geq 40 \mathrm{~g} /$ day on the highfibre diet intervention and the difference in fibre intake between interventions ( $\Delta$ fibre) of $>25 \mathrm{~g} /$ day. Macronutrient profile and study design variously modified effects in each analysis, with no clear patterns.

Multivariate analyses To explore the heterogeneity identified in univariate analyses, inverse variance weighted multiple regression models assessed the independent predictors of effect for outcomes in the full database combining all three pooled analyses (ESM Table 5). The models were significant $(p<0.05)$ for FBG and FBI only, explaining $47 \%$ and $66 \%$ of the variation in these outcomes, respectively. Significant $(p<0.05)$ independent predictors from strongest to weakest were identified as dietary carbohydrate $(\% \mathrm{E})(\beta=-0.034$, partial $R^{2}$ [adjusted for other variables]=0.22), type 2 diabetes $\left(\beta=-0.57\right.$, partial $\left.R^{2}=0.18\right)$, pulses alone interventions $\left(\beta=-0.71\right.$, partial $\left.R^{2}=0.18\right)$, and dietary protein $(\% \mathrm{E})$ $\left(\beta=0.084\right.$, partial $\left.R^{2}=0.14\right)$ for $\mathrm{FBG}$; metabolic feeding control $\left(\beta=1.05\right.$, partial $\left.R^{2}=0.41\right)$ and $\Delta$ fibre $(\beta=0.056$, partial $R^{2}=0.39$ ) for $\mathrm{FBI}$; and pulses alone interventions $\left(\beta=0.92\right.$, partial $\left.R^{2}=0.31\right)$ and follow-up (days) $(\beta=-0.0046$, partial $R^{2}=0.23$ ) for GP.

Publication bias Funnel plots for each outcome were inspected for the presence of publication bias in the full database combining all three analyses (ESM Fig. 1). There was limited evidence of funnel plot asymmetry for FBG and FBI with a tendency for publication of small trials reporting large effects and error estimates that favour pulses (three trials for FBG and one for FBI) but no small trials reporting large effects that favour control. No evidence of asymmetry was observed for HOMA-IR or GP.

\section{Discussion}

The present three pooled analyses of 41 randomised controlled experimental trials, in a total of 1,674 participants, are supportive of diabetes association nutrition guidelines recommending the consumption of dietary pulses 


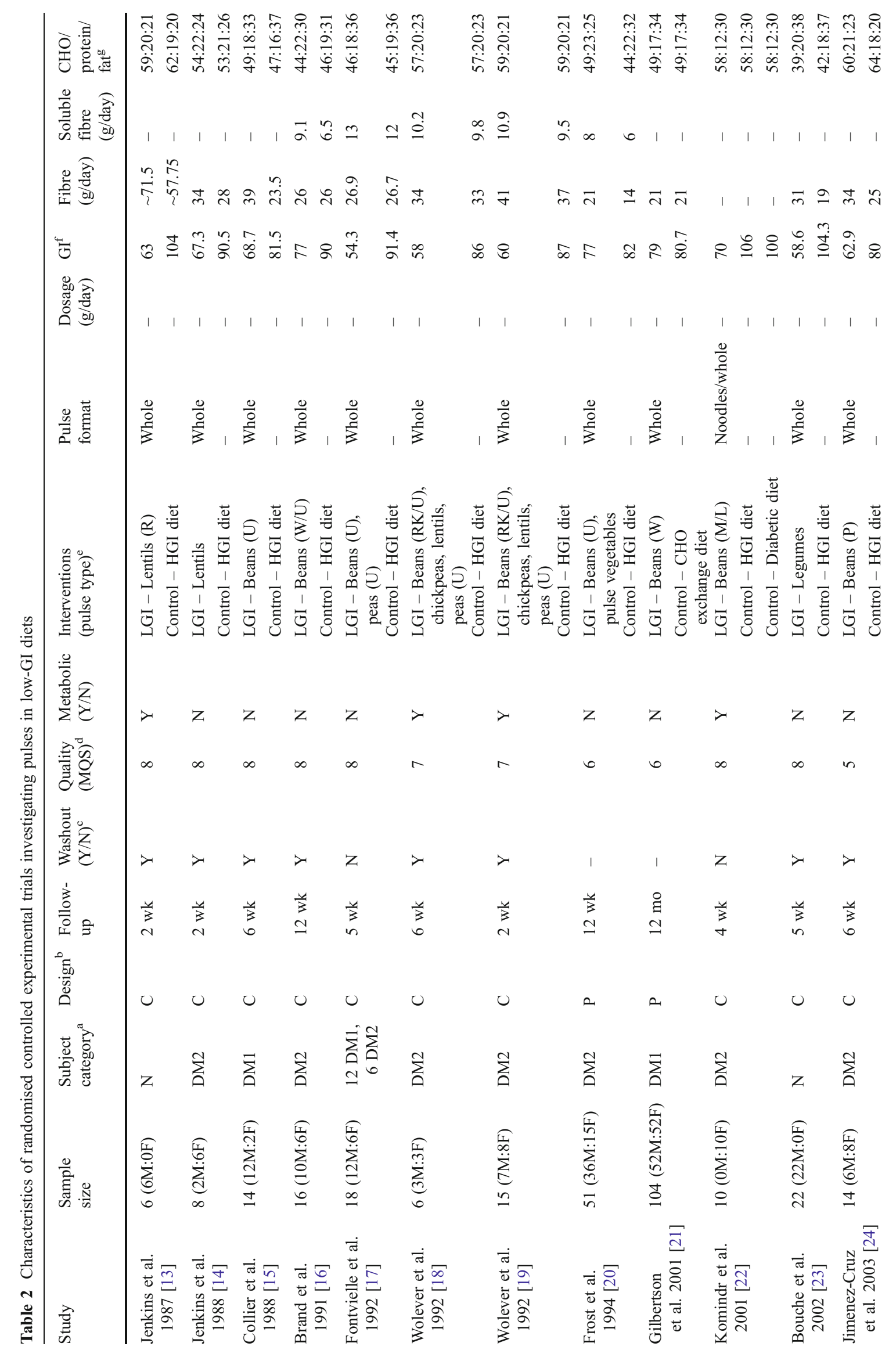




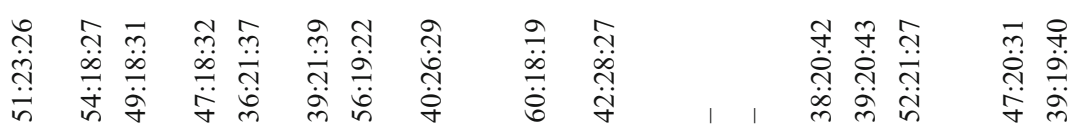

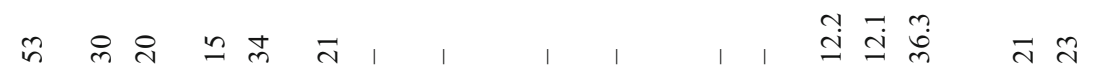

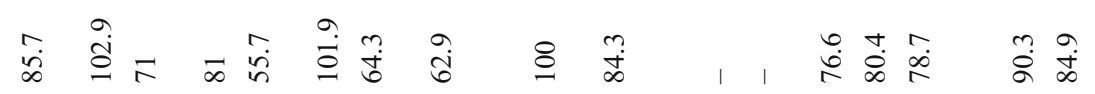

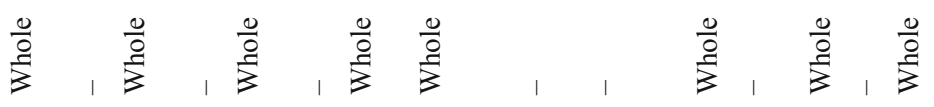

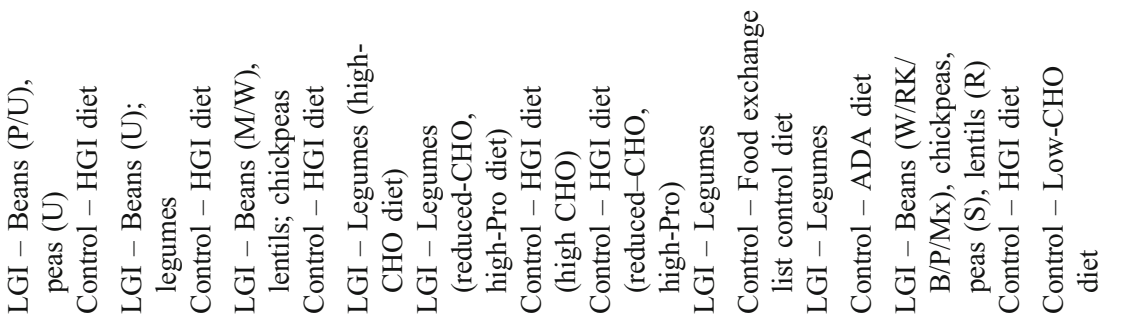

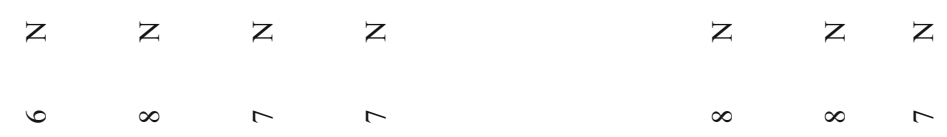

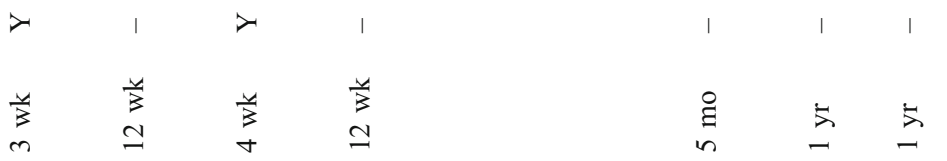

o 2 o 2

20

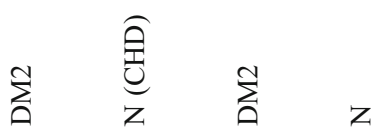

$\sum^{1} \sum^{N} \sum^{2}$

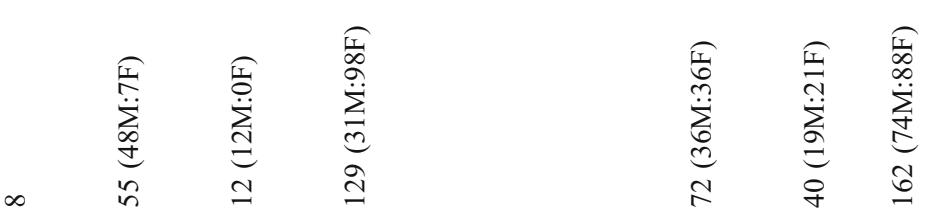

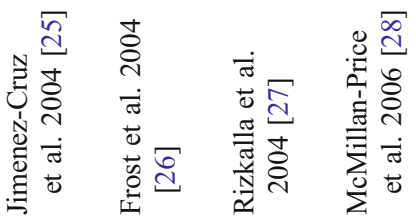

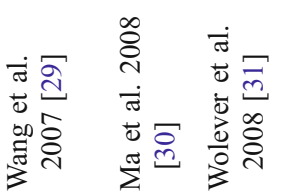

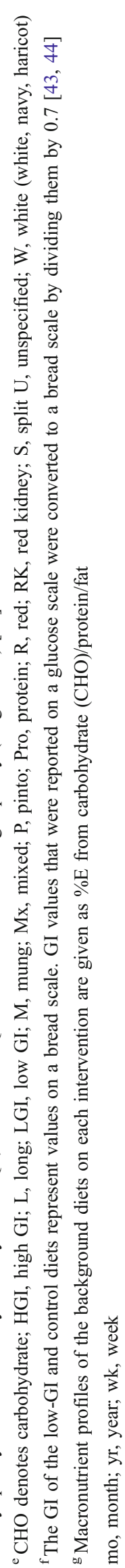




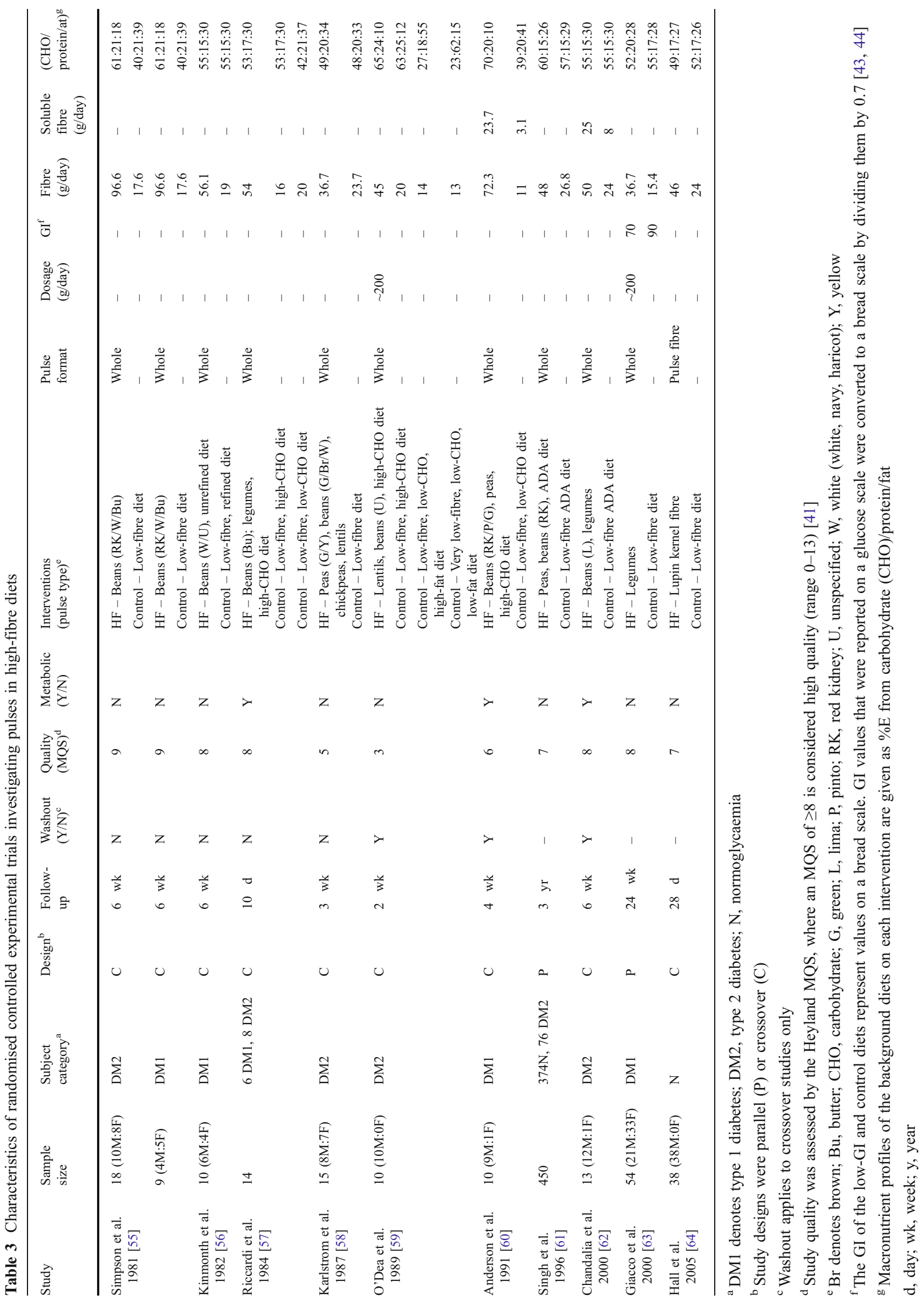


Fig. 2 Pooled effect of interventions involving pulses alone on the following markers of glycaemic control: FBG (a), FBI (b), HOMA-IR (c) and GP (d). SMDs (boxes) with 95\% CIs (lines) for each study, study weights represented by the size of each box, and pooled effect estimates (diamonds) with $95 \%$ CI represented by the width of the diamonds were generated using the general inverse variance method with random (FBG, FBI, HOMA-IR) and fixed (GP) effects models. The article by Jang et al. 2001 [50] reported two separately controlled trials, one denoted as DM2 in patients with type 2 diabetes and coronary artery disease, and one denoted as $\mathrm{CAD}$ in patients with coronary artery disease alone a

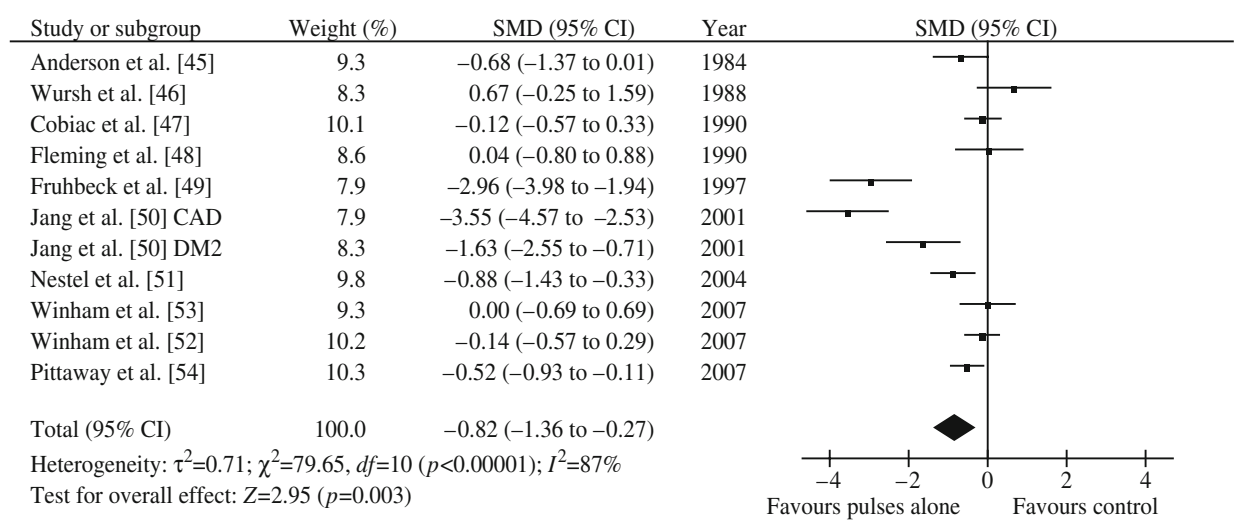

b

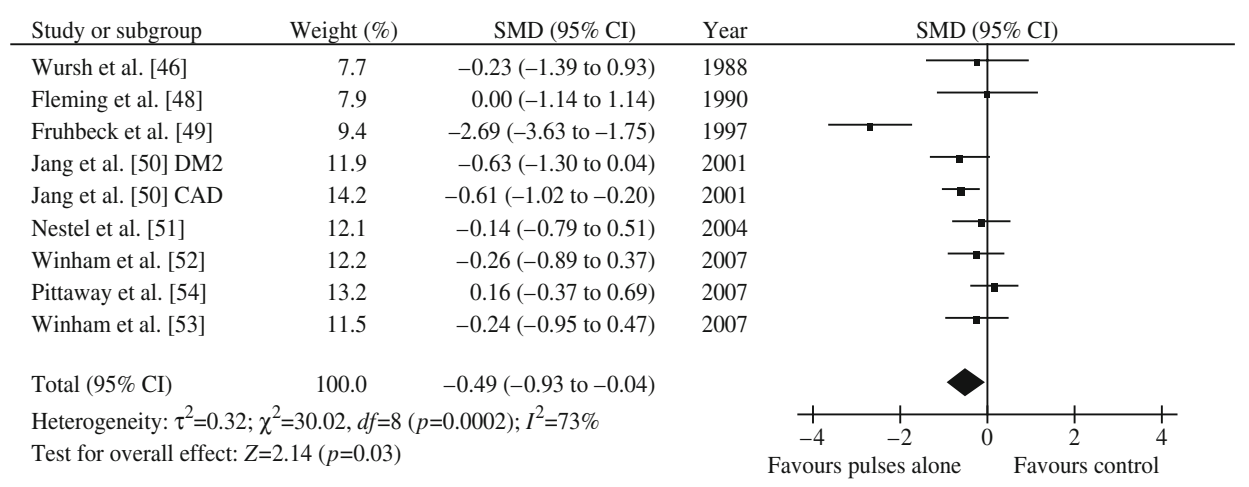

C

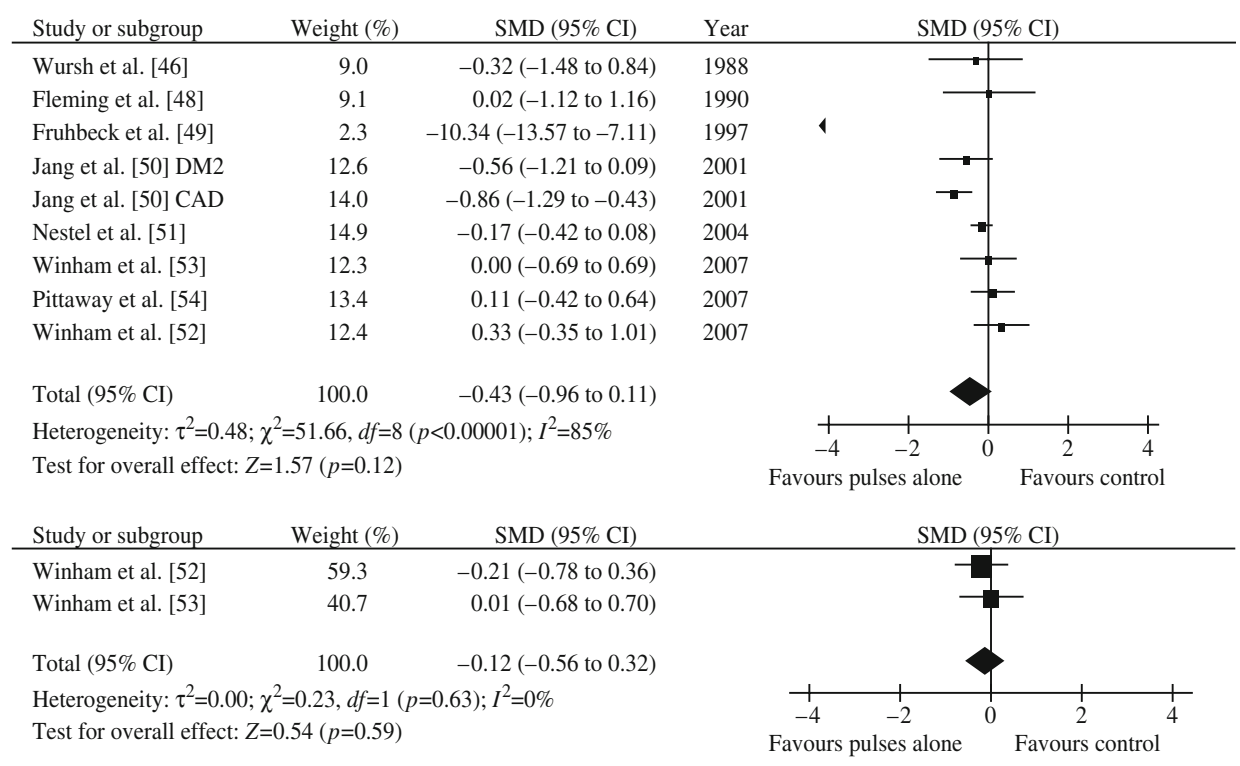

as a means of optimising diabetes control. Pulses given alone or as part of low-GI or high-fibre diets were found to improve the main markers of long-term glycaemic control, namely, GP ( $\mathrm{HbA}_{1 \mathrm{c}}$ or fructosamine) by pulses in low-GI and high-fibre diets and FBG by pulses given alone or as part of high-fibre diets. The magnitude of the benefit conferred in terms of the normalisation of $\mathrm{HbA}_{1 \mathrm{c}}$ approached the $0.58-0.77 \%$ reduction reported for acarbose in two recent meta-analyses in type 2 diabetes [8, 65], which was found to be related to reduced cardiovascular events [8]. If we calculate the mean absolute reduction in $\mathrm{HbA}_{1 \mathrm{c}}$ in people with type 2 diabetes in the present analysis (mean difference $\mathrm{HbA}_{1 \mathrm{c}}=\operatorname{SMDGP} \times$ pooled $\mathrm{SD} \mathrm{HbA}_{1 \mathrm{c}}$ ), then the SMD reductions in GP for pulses in low-GI and high-fibre diets correspond to an absolute reduction in $\mathrm{HbA}_{1 \mathrm{c}}$ of $\sim 0.48 \%$, a reduction that exceeds the clinically meaningful threshold $(\geq 0.3 \%$ ) proposed by the US Food and Drug Administration [66] and lies at the lower limit of efficacy expected for oral agents [1]. Observed benefits, however, were complicated by significant inter-study heterogeneity.

Possible modifiers of the primary effects were explored by planned and post hoc subgroup analyses, which 
Fig. 3 Pooled effect of pulses in low-GI (LGI) diets on the following markers of glycaemic control: FBG (a), FBI (b),

HOMA-IR (c) and GP (d) SMDs (boxes) with 95\% CIs (lines) for each study, study weights represented by the size of each box, and pooled effect estimates (diamonds) with $95 \%$ CI represented by the width of the diamonds were generated using the general inverse variance method with random (FBG, HOMA-IR, GP) and fixed (FBI) effects models. The article by McMillan-Price et al. 2006 [28] reported two separately controlled trials, one denoted as $\mathrm{HC}$, a highcarbohydrate intervention, and one denoted as HP, a high protein intervention a

\begin{tabular}{|c|c|c|}
\hline Study or subgroup & Weight $(\%)$ & $\mathrm{SMD}(95 \% \mathrm{CI})$ \\
\hline Jenkins et al. [13] & 7.7 & $0.61(0.34$ to 0.88$)$ \\
\hline Collier et al. [15] & 3.2 & $0.52(-0.36$ to 1.40$)$ \\
\hline Jenkins et al. [14] & 2.8 & $-1.08(-2.06$ to -0.10$)$ \\
\hline Brand et al. [16] & 5.3 & $0.07(-0.48$ to 0.62$)$ \\
\hline Fontvielle et al. [17] & 5.6 & $-0.16(-0.67$ to 0.35$)$ \\
\hline Wolever et al. [18] & 5.1 & $0.00(-0.57$ to 0.57$)$ \\
\hline Wolever et al. [19] & 3.1 & $-0.20(-1.10$ to 0.70$)$ \\
\hline Frost et al. [20] & 6.6 & $-0.26(-0.65$ to 0.13$)$ \\
\hline Bouche et al. [23] & 4.4 & $0.25(-0.42$ to 0.92$)$ \\
\hline Jimenez-Cruz et al. [24] & 8.3 & $-0.35(-0.55$ to -0.15$)$ \\
\hline Jimenez-Cruz et al. [25] & 2.8 & $-1.11(-2.09$ to -0.13$)$ \\
\hline Rizkalla et al. [27] & 3.4 & $-1.10(-1.94$ to -0.26$)$ \\
\hline Frost et al. [26] & 6.6 & $0.41(0.02$ to 0.80$)$ \\
\hline McMillan-Price et al. [28] HC & 7.0 & $-0.04(-0.39$ to 0.31$)$ \\
\hline McMillan-Price et al. [28] HP & 7.0 & $0.14(-0.21$ to 0.49$)$ \\
\hline Wang et al. [29] & 7.2 & $-0.29(-0.62$ to 0.04$)$ \\
\hline Ma et al. [30] & 6.3 & $0.00(-0.43$ to 0.43$)$ \\
\hline Wolever et al. [31] & 7.7 & $0.14(-0.13$ to 0.41$)$ \\
\hline Total $(95 \% \mathrm{CI})$ & 100.0 & $-0.06(-0.25$ to 0.14$)$ \\
\hline
\end{tabular}

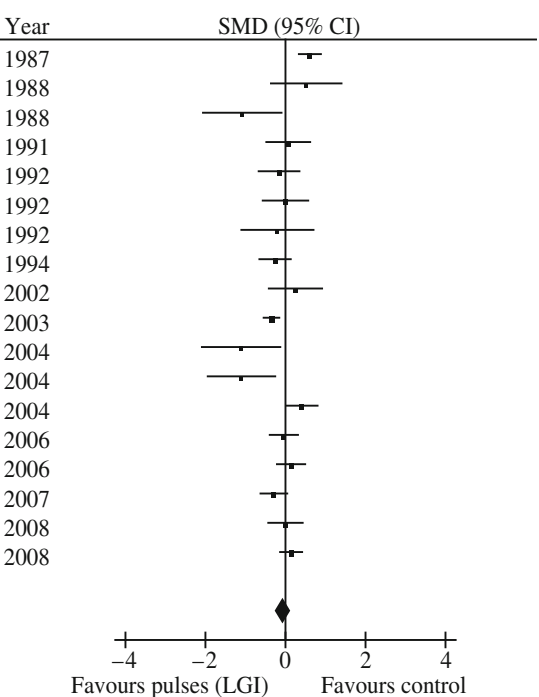

b Test for overall effect: $Z=0.57(p=0.57)$

Favours pulses (LGI) Favours control

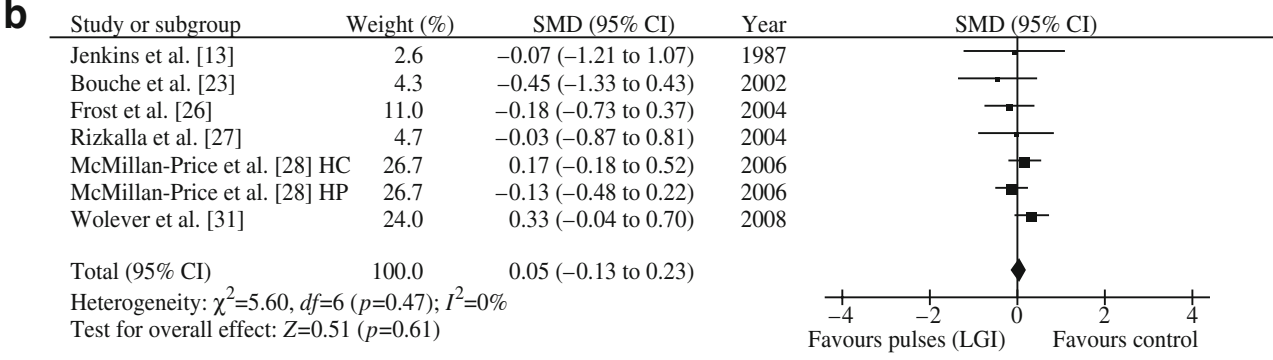

C

\begin{tabular}{lcccc} 
Study or subgroup & Weight $(\%)$ & SMD $(95 \%$ CI $)$ & Year & SMD (95\% CI) \\
\hline Jenkins et al. [13] & 7.8 & $0.34(-0.82$ to 1.50$)$ & 1987 \\
Bouche et al. [23] & 7.4 & $-1.46(-2.66$ to -0.26$)$ & 2002 \\
Frost et al. [26] & 19.8 & $0.22(-0.33$ to 0.77$)$ & 2004 \\
Rizkalla et al. [27] & 11.6 & $-0.48(-1.36$ to 0.40$)$ & 2004 \\
McMillan-Price et al. [28] HP & 26.7 & $0.26(-0.09$ to 0.61$)$ & 2006 \\
McMillan-Price et al. [28] HC & 26.7 & $-0.18(-0.53$ to 0.17$)$ & 2006 \\
& 100.0 & $-0.07(-0.44$ to 0.29$)$ & & \\
Total (95\% CI) & & & Favours pulses (LGI) Favours control
\end{tabular}

d

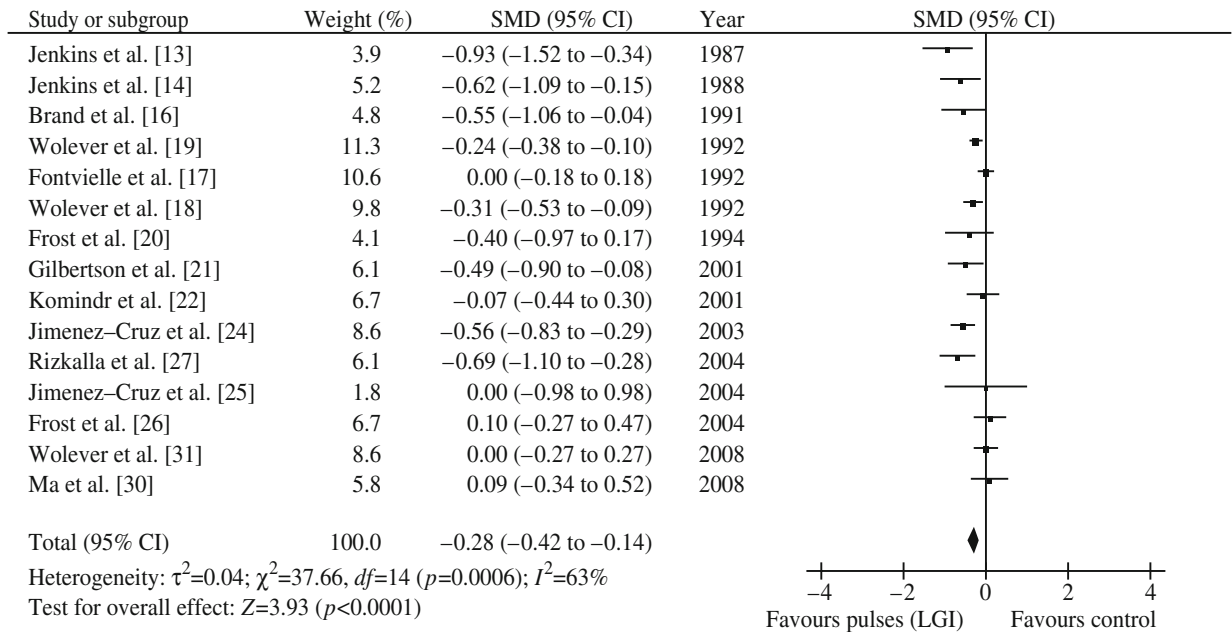

identified several conditions under which benefits were gained, maintained or lost, despite the heterogeneity remaining largely unexplained. Diabetes status was found to be one modifier. A benefit of pulses for FBG and/or GP was seen in either type 2 diabetes or any diabetes for all three pooled analyses. In multiple regression models, type 2 diabetes was identified as an independent predictor of FBG, explaining $26 \%$ of the variation in this outcome. In 
Fig. 4 Pooled effect of pulses in high-fibre (HF) diets on the following markers of glycaemic control: FBG (a), FBI (b),

HOMA-IR (c) and GP (d) SMDs (boxes) with 95\% CIs (lines) for each study, study weights represented by the size of each box, and pooled effect estimates (diamonds) with $95 \%$ CI represented by the width of the diamonds were generated using the general inverse variance method with random (FBG, GP) and fixed (FBI, HOMA-IR) effects models. The article by Simpson et al. 1981 [55] reported two separately controlled trials, one denoted as DM1 in patients with type 1 diabetes, and one denoted as DM2 in type 2 diabetes a

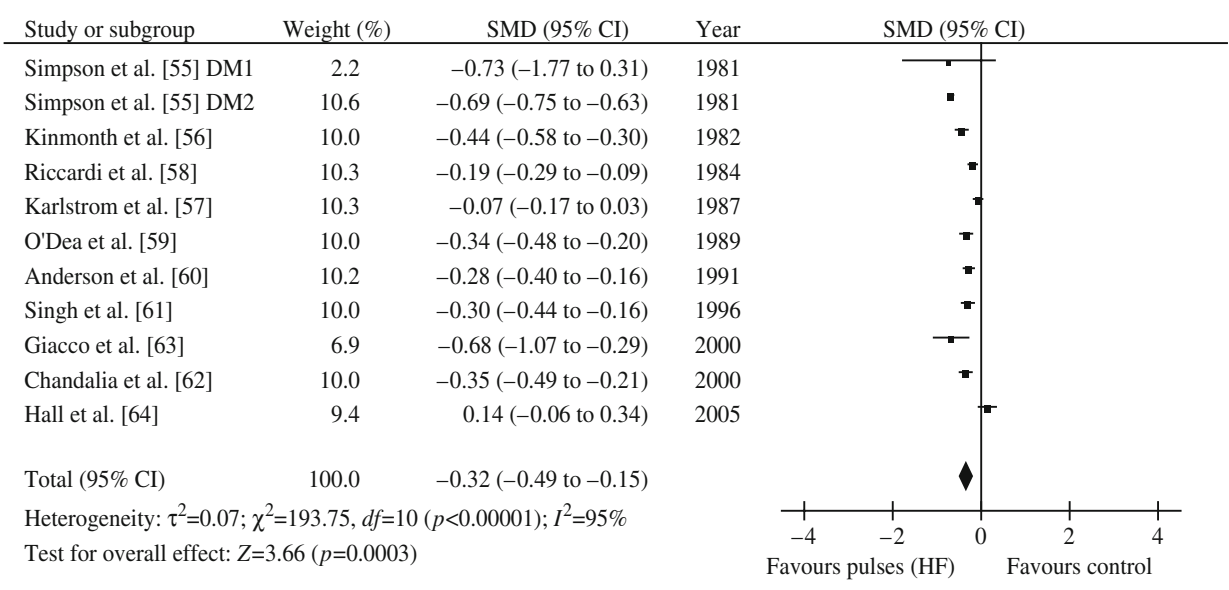

b

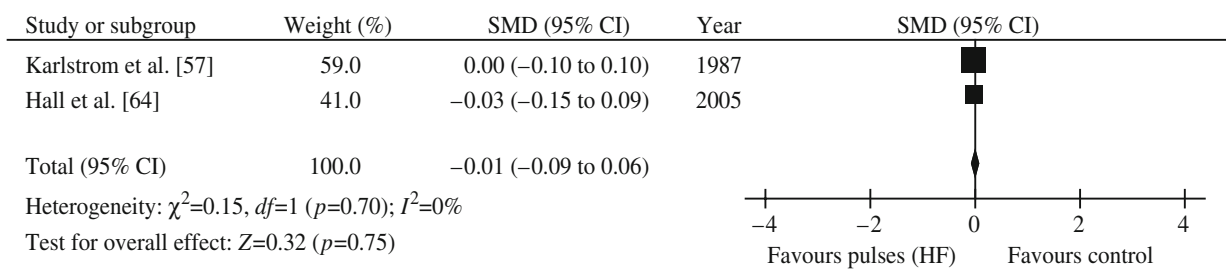

C

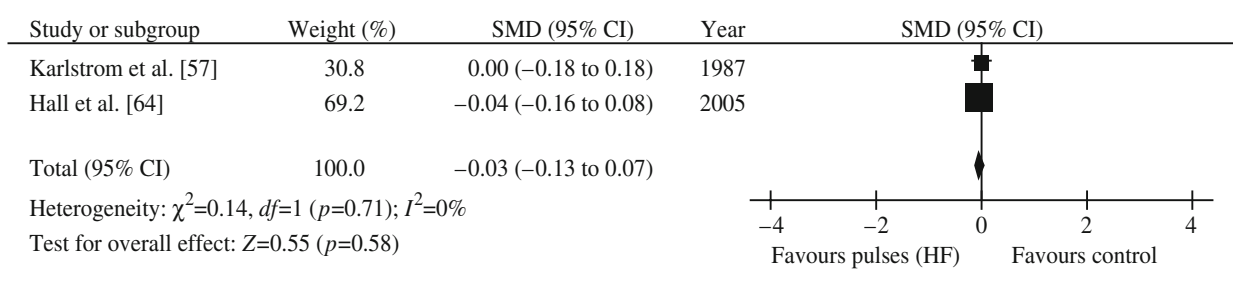

d

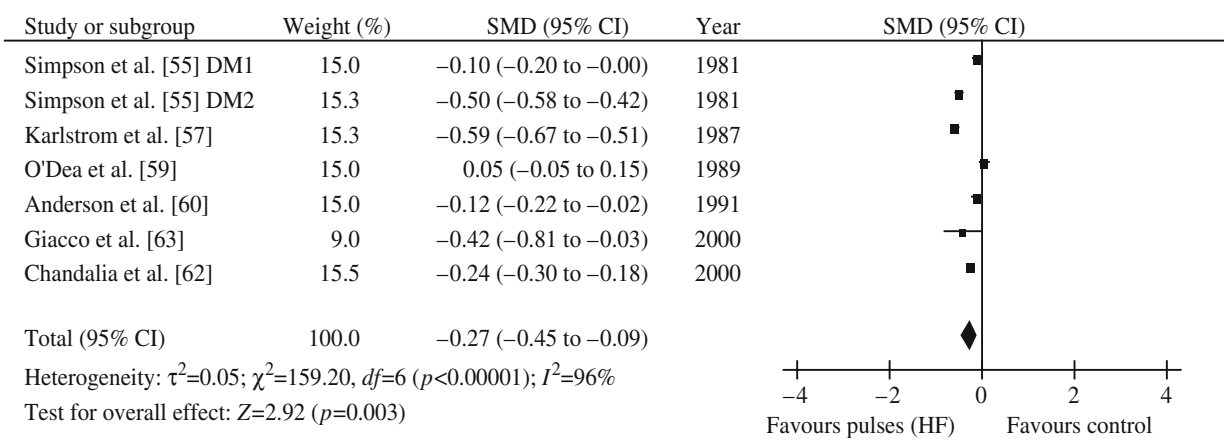

contrast, the data in healthy normoglycaemic individuals were conflicting. There was a decreasing effect of pulses alone on FBG in the analysis of pulses alone, whereas there was an increasing effect of pulses in low-GI diets on FBG in healthy normoglycaemic individuals. Heterogeneity was reduced only for type 1 diabetes, remaining substantial to considerable across other categories of participants.

Glycaemic benefits also appeared to be modified by pulse type. The strongest evidence for benefit was for pulse interventions containing chickpeas (Cicer arietunum), which decreased FBG in the pulses alone analysis with very low heterogeneity. Potential benefit was also seen for
Phaseolus vulgaris (black, white, pinto, red and white kidney beans), but interpretation of these results was complicated by high heterogeneity, and for Vicia faba (fava beans), but the data were from only one study. Disentangling an effect of pulse type was made difficult by differences in physical form. In the analysis of pulses alone, the benefit of pulses was significant only for pulses as flakes/flour for FBI and HOMA-IR, and the effect estimates were much larger in favour of pulses as flakes/flour for FBG. In contrast, benefit was seen only for whole pulses in the analysis of pulses in high-fibre diets. Univariate and multivariate analyses of the effect of pulse type in low-GI diets and high-fibre diets were 
not undertaken owing to confounding from deficiencies in reporting and the simultaneous use of multiple pulse types. More trials are needed to define optimal pulse types and physical form.

Another possible modifier of benefit was pulse dose. Benefits of pulses alone for FBG and FBI and HOMA-IR were unexpectedly seen only at doses less than the serving recommended by the American Heart Association (1/2 cup/ day $\approx 100 \mathrm{~g} /$ day) [42]. Although the interactive effects of pulse dose could not be assessed in the analyses of pulses in low-GI and high-fibre diets, the level of dietary GI or dietary fibre achieved during the trials can be considered as proxies for the dose. If we take this approach, then only in the context of trials that achieved a true low-GI intervention $(\mathrm{GI}<70)$ or large GI treatment differences $(\Delta \mathrm{GI}>15)$ did pulses in low-GI diets decrease GP and show a tendency for lowering FBG. Similarly, only at fibre intakes at the EASD recommended level ( $>40 \mathrm{~g} /$ day) [32], did pulses in highfibre diets decrease FBG. The difference in fibre intake between the pulse and control interventions ( $\Delta$ fibre), however, was identified as a positive independent predictor of FBI, explaining $40 \%$ of the variation in this outcome in multiple regression models. These discrepancies in the data for dose and its proxies need to be reconciled by welldesigned, long-term dose-response trials.

Follow-up was a further possible modifier. The benefit of pulses alone for FBG and FBI and of pulses in high-fibre diets for GP was only seen when the duration of follow-up was $>4$ weeks. Follow-up was identified as a negative independent predictor of GP, explaining $23 \%$ of the variation in this outcome in multiple regression models. These data suggest that an adaptation period may be needed to realise the benefits of pulses. Trials with sufficiently long-term follow-up are needed to confirm the sustainability of benefits.

Other modifiers may have related to aspects of the macronutrient profile of background diets. Although macronutrient profile was not a reliable modifier of the effect of pulses in univariate subgroup analyses with both high and low carbohydrate $(<$ and $\geq 55 \% \mathrm{E})$, fat $(\leq$ and $>30 \% \mathrm{E})$ and protein $(<$ and $\geq 20 \% \mathrm{E}$ ) diets variously modifying the effect of pulses in the pulses in low-GI and high-fibre diets analyses, both carbohydrate and protein were identified as independent predictors of FBG in multiple regression models. Dietary carbohydrate was inversely associated with FBG, explaining $22 \%$ of its variation, whereas protein was positively associated with FBG, explaining $14 \%$ of its variation.

Finally, aspects of study design and quality were identified as potential modifiers of benefit. Although a crossover or parallel design itself was not a reliable modifier of the effect of pulses, benefit was only seen for crossover trials with washout periods for FBG in the pulses in high-fibre diets analysis and GP in the pulses in the lowGI diets analysis. Metabolic feeding control also appeared to be driving some of the effects. Benefit was only seen in trials with metabolic feeding control for GP in the pulses in low-GI diets analysis and both FBG and GP in the pulses in high-fibre diets analysis. It was also identified as an independent predictor of FBI in multiple regression models, explaining $42 \%$ of the variation in this outcome. Overall methodological quality of the trials appeared to exert a further influence. Only trials that that did not meet the Heyland criteria for high quality $(\mathrm{MQS}<8)$ [41] exerted a benefit in terms of FBG and GP, with high heterogeneity observed in all three pooled analyses. There was one exception for the effect on GP in the analysis of pulses in high-fibre diets, where the opposite was true. MQS, however, was not identified as an independent predictor for any outcome in multiple regression models. To address the effect of designs and quality on reproducibility, these data suggest the need for high-quality, 'real world' effectiveness (non-metabolic) trials with careful measures of compliance, side effects and acceptability, and designs to eliminate potential sequence and carryover effects.

Our data support the proposed main mechanism of action of pulses. Data from acute and chronic feeding studies suggest that pulses reduce both postprandial blood glucose and insulin excursions [46, 48, 50, 51], effectively lowering the GI of the diet through slowed absorption [11, $67,68]$. A direct benefit on measures of insulin sensitivity has also been reported for pulses alone using surrogate HOMA indices $[50,51]$ and has been implied by studies of pulses in low-GI diets using the euglycaemic-hyperinsulinaemic clamp [27]. The significant decrease in FBI in the presence of decreased FBG seen in the pooled analysis of pulses alone suggests an insulin-sensitising effect of pulses. However, this combination did not translate into a significant reduction in HOMA-IR, although the direction of the effect favoured pulses. These markers pertain only to the fasting state and are considered to be more closely related to hepatic insulin sensitivity than whole body insulin sensitivity [69]. It is more likely that postprandial measures of insulin sensitivity would show a benefit. This suggestion is supported by the larger effect sizes seen for insulin reduction in post-oralglucose or mixed-meal profiles (120-360 min) following chronic feeding of pulses alone [46, 48, 50, 51]. These proposed mechanisms, however, need to be confirmed by euglycaemic-hyperinsulinaemic clamp studies.

Several caveats of our analysis need to be considered. First, our pooled analyses did not include long-term, postprandial glycaemic control outcomes. Although numerous studies showing acute, postprandial [44] and 'second meal' effects [68] can be found in the literature, there were only a handful of trials reporting long-term, postprandial data in each pooled analysis, and these data were derived from a 
variety of diverse protocols, precluding meta-analyses. As postprandial glycaemic outcomes tend to be more sensitive markers of glycaemic control than are fasting outcomes [70], it can be argued that the effect estimates may have been larger, and thus more likely to be significant, with possibly less inter-study heterogeneity. Second, only published studies were included in the present analyses, making a publication bias possible. Funnel plots for the pooled analysis of pulses alone showed particular evidence of possible bias favouring the publication of small, positive trials with large effect and error estimates. Finally, heterogeneity remained unsatisfactorily explained by univariate subgroup analyses in the three pooled analyses, compromising the quality of the evidence. Some steps were taken to address this limitation. Exploratory multiple regression models were used to explain $>45 \%$ of the variability in FBG and FBI and to identify significant independent predictors for GP, although we readily acknowledge the serious limitations in performing analyses with so few observations (trials). Random effects models were also used to incorporate the heterogeneity into the analyses, and systematic removal of each study during sensitivity analyses did not alter conclusions.

In conclusion, dietary non-oil-seed pulses may modestly improve medium to longer term glycaemic control through a possible insulin-sparing mechanism, when used alone or in combination with other dietary interventions to lower the GI or increase the dietary fibre of the diet. This benefit appears to be especially true when the pulse type is chickpeas, individuals have diabetes, diets are metabolically controlled, or the duration of follow-up is $>4$ weeks, conditions for which the best evidence exists. Although these factors do not offer robust explanations for the high level of heterogeneity, a benefit of pulses added to the diets of individuals with diabetes cannot be excluded. The heterogeneity in the pooled data highlight the need for large, well-designed, carefully conducted randomised controlled trials to resolve issues raised by the subgroup analyses of differential benefits related to diabetes status, pulse type, dose, physical form, follow-up, study quality, macronutrient profile of background diets, feeding control and design. A broader benefit of dietary pulses on metabolism must also be considered. Future analyses are planned to assess the effect of pulses on other areas of metabolic control, including lipids, body weight and blood pressure. The effect of pulses in other dietary interventions, such as Mediterranean or vegetarian diets, also needs to be studied.

Acknowledgements Aspects of this work were presented in abstract form at the 7th International Symposium on Multiple Risk Factors in Cardiovascular Disease, Venice, Italy, 22-25 October 2008 [71] and Experimental Biology 2009, New Orleans, LA, USA, 18-22 April 2009 [72]. Research and travel funding were provided by Pulse Canada (Winnipeg, MB, Canada). J. L. Sievenpiper was funded by a Province of Ontario Postdoctoral Fellowship, the Edie Steinberg
Scholarship Fund, and the Edward Christie Stevens Fellowship in Medicine. J. M. W. Wong was funded by a Canadian Institutes of Health Research (CIHR) Doctoral Research Award. D. J. A. Jenkins was funded by the Government of Canada through the Canada Research Chair Endowment.

Duality of interest (within last 12 months) J. L. Sievenpiper, C. W. C. Kendall and R. P. Bazinet have received consultant fees from Pulse Canada via BDSK Consulting (Toronto, ON, Canada). C. W. C. Kendall also serves on the scientific advisory board for Pulse Canada and has served on the scientific advisory board, received research support, travel support, consultant fees or honoraria from Barilla, Solae, Unilever, Haine Celestial, Loblaws, Oldways Preservation Trust, the Almond Board of California, the International Nut Council, Paramount Farms, the California Strawberry Commission, and the Canola and Flax Councils of Canada. A. Esfahani, J. M. W. Wong, A. J. Carleton, H. Y. Jiang and E. Vidgen have no declared duality of interest. D. J. A. Jenkins has served on the scientific advisory board for or received research support, consultant fees, or honoraria from Barilla, Solae, Unilever, Haine Celestial, Loblaws, Sanitarium Company, Herbalife International, Pacific Health Laboratories, Metagenics/MetaProteomics, Bayer Consumer Care, Oldways Preservation Trust, The Almond Board of California, the California Strawberry Commission, Orafti, and the Canola and Flax Councils of Canada.

\section{References}

1. Inzucchi SE (2002) Oral antihyperglycemic therapy for type 2 diabetes: scientific review. JAMA 287:360-372

2. Nathan DM (2006) Thiazolidinediones for initial treatment of type 2 diabetes? N Engl J Med 355:2477-2480

3. Gan D (ed) (2003) Diabetes Atlas, 2nd edn. International Diabetes Federation, Brussels

4. Gerstein HC, Miller ME, Byington RP et al (2008) Effects of intensive glucose lowering in type 2 diabetes. $\mathrm{N}$ Engl $\mathrm{J}$ Med 358:2545-2559

5. Nissen SE, Wolski K (2007) Effect of rosiglitazone on the risk of myocardial infarction and death from cardiovascular causes. N Engl J Med 356:2457-2471

6. Patel A, MacMahon S, Chalmers J et al (2008) Intensive blood glucose control and vascular outcomes in patients with type 2 diabetes. N Engl J Med 358:2560-2572

7. UK Prospective Diabetes Study (UKPDS) Group (1998) Effect of intensive blood-glucose control with metformin on complications in overweight patients with type 2 diabetes (UKPDS 34). Lancet 352:854-865

8. Hanefeld M, Cagatay M, Petrowitsch T, Neuser D, Petzinna D, Rupp M (2004) Acarbose reduces the risk for myocardial infarction in type 2 diabetic patients: meta-analysis of seven long-term studies. Eur Heart J 25:10-16

9. Chiasson JL, Josse RG, Gomis R, Hanefeld M, Karasik A, Laakso M (2002) Acarbose for prevention of type 2 diabetes mellitus: the STOP-NIDDM randomised trial. Lancet 359:2072-2077

10. Tormo MA, Gil-Exojo I, Romero de Tejada A, Campillo JE (2004) Hypoglycaemic and anorexigenic activities of an alphaamylase inhibitor from white kidney beans (Phaseolus vulgaris) in Wistar rats. Br J Nutr 92:785-790

11. Jenkins DJ, Wolever TM, Taylor RH, Barker HM, Fielden H (1980) Exceptionally low blood glucose response to dried beans: comparison with other carbohydrate foods. BMJ 281:578-580

12. Jenkins DJ, Ghafari H, Wolever TM et al (1982) Relationship between rate of digestion of foods and post-prandial glycaemia. Diabetologia 22:450-455 
13. Jenkins DJ, Wolever TM, Collier GR et al (1987) Metabolic effects of a low-glycemic-index diet. Am J Clin Nutr 46:968-975

14. Jenkins DJ, Wolever TM, Buckley G et al (1988) Low-glycemicindex starchy foods in the diabetic diet. Am J Clin Nutr 48:248-254

15. Collier GR, Giudici S, Kalmusky J et al (1988) Low glycaemic index starchy foods improve glucose control and lower serum cholesterol in diabetic children. Diabetes Nutr Metab 1:11-18

16. Brand JC, Colagiuri S, Crossman S, Allen A, Roberts DC, Truswell AS (1991) Low-glycemic index foods improve longterm glycemic control in NIDDM. Diabetes Care 14:95-101

17. Fontvieille AM, Rizkalla SW, Penfornis A, Acosta M, Bornet FR, Slama G (1992) The use of low glycaemic index foods improves metabolic control of diabetic patients over five weeks. Diabet Med 9:444-450

18. Wolever TM, Jenkins DJ, Vuksan V, Jenkins AL, Wong GS, Josse RG (1992) Beneficial effect of low-glycemic index diet in overweight NIDDM subjects. Diabetes Care 15:562-564

19. Wolever TM, Jenkins DJ, Vuksan V et al (1992) Beneficial effect of a low glycaemic index diet in type 2 diabetes. Diabet Med 9:451-458

20. Frost G, Wilding J, Beecham J (1994) Dietary advice based on the glycaemic index improves dietary profile and metabolic control in type 2 diabetic patients. Diabet Med 11:397-401

21. Gilbertson HR, Brand-Miller JC, Thorburn AW, Evans S, Chondros P, Werther GA (2001) The effect of flexible low glycemic index dietary advice vs measured carbohydrate exchange diets on glycemic control in children with type 1 diabetes. Diabetes Care 24:1137-1143

22. Komindr S, Ingsriswang S, Lerdvuthisopon N, Boontawee A (2001) Effect of long-term intake of Asian food with different glycemic indices on diabetic control and protein conservation in type 2 diabetic patients. J Med Assoc Thai 84:85-97

23. Bouche C, Rizkalla SW, Luo J et al (2002) Five-week, lowglycemic index diet decreases total fat mass and improves plasma lipid profile in moderately overweight nondiabetic men. Diabetes Care 25:822-828

24. Jimenez-Cruz A, Bacardi-Gascon M, Turnbull WH, RosalesGaray P, Severino-Lugo I (2003) A flexible, low-glycemic index Mexican-style diet in overweight and obese subjects with type 2 diabetes improves metabolic parameters during a 6-week treatment period. Diabetes Care 26:1967-1970

25. Jimenez-Cruz A, Turnbull WH, Bacardi-Gascon M, RosalesGaray P (2004) A high-fiber, moderate-glycemic-index, Mexican style diet improves dyslipidemia in individuals with type 2 diabetes. Nutr Res 24:19-27

26. Frost GS, Brynes AE, Bovill-Taylor C, Dornhorst A (2004) A prospective randomised trial to determine the efficacy of a low glycaemic index diet given in addition to healthy eating and weight loss advice in patients with coronary heart disease. Eur J Clin Nutr 58:121-127

27. Rizkalla SW, Taghrid L, Laromiguiere M et al (2004) Improved plasma glucose control, whole-body glucose utilization, and lipid profile on a low-glycemic index diet in type 2 diabetic men: a randomized controlled trial. Diabetes Care 27:1866-1872

28. McMillan-Price J, Petocz P, Atkinson F et al (2006) Comparison of 4 diets of varying glycemic load on weight loss and cardiovascular risk reduction in overweight and obese young adults: a randomized controlled trial. Arch Intern Med 166:1466-1475

29. Wang H, Yang Y, Zhang Y, Han J, Wang Z (2007) Acceptance to knowledge to food glycemic index and dietary adjustment in diabetic patients. J Clin Rehabil Tissue Eng Res 11:10701-10703

30. Ma Y, Olendzki BC, Merriam PA et al (2008) A randomized clinical trial comparing low-glycemic index vs ADA dietary education among individuals with type 2 diabetes. Nutrition 24:45-56

31. Wolever TM, Gibbs AL, Mehling C et al (2008) The Canadian Trial of Carbohydrates in Diabetes (CCD), a 1-y controlled trial of low-glycemic-index dietary carbohydrate in type 2 diabetes: no effect on glycated hemoglobin but reduction in C-reactive protein. Am J Clin Nutr 87:114-125

32. Mann JI, De Leeuw I, Hermansen K et al (2004) Evidence-based nutritional approaches to the treatment and prevention of diabetes mellitus. Nutr Metab Cardiovasc Dis 14:373-394

33. CDACPGE Committee (2008) Canadian Diabetes Association 2008 clinical practice guidelines for the prevention and management of diabetes in Canada. Can J Diabetes 32(Suppl 1):S1-S201

34. Bantle JP, Wylie-Rosett J, Albright AL et al (2008) Nutrition recommendations and interventions for diabetes: a position statement of the American Diabetes Association. Diabetes Care 31(Suppl 1):S61-S78

35. Higgins JPT, Green S (eds) (2008) Cochrane handbook for systematic reviews of interventions version 5.0.0 [updated February 2008]. The Cochrane Collaboration. Available from www.cochrane-handbook.org, accessed 24 April, 2009

36. Moher D, Cook DJ, Eastwood S, Olkin I, Rennie D, Stroup DF (1999) Improving the quality of reports of meta-analyses of randomised controlled trials: the QUOROM statement. Quality of reporting of meta-analyses. Lancet 354:1896-1900

37. Furukawa TA, Barbui C, Cipriani A, Brambilla P, Watanabe $N$ (2006) Imputing missing standard deviations in meta-analyses can provide accurate results. J Clin Epidemiol 59:7-10

38. Elbourne DR, Altman DG, Higgins JP, Curtin F, Worthington HV, Vail A (2002) Meta-analyses involving cross-over trials: methodological issues. Int J Epidemiol 31:140-149

39. Matthews DR, Hosker JP, Rudenski AS, Naylor BA, Treacher DF, Turner RC (1985) Homeostasis model assessment: insulin resistance and beta-cell function from fasting plasma glucose and insulin concentrations in man. Diabetologia 28:412-419

40. NIST/SEMATECH (2006) NIST/SEMATECH e-Handbook of Statistical Methods. Available from http://www.itl.nist.gov/div898/ handbook/, accessed 24 April, 2009

41. Heyland DK, Novak F, Drover JW, Jain M, Su X, Suchner U (2001) Should immunonutrition become routine in critically ill patients? A systematic review of the evidence. JAMA 286: 944-953

42. Lichtenstein AH, Appel LJ, Brands M et al (2006) Diet and lifestyle recommendations revision 2006: a scientific statement from the American Heart Association Nutrition Committee. Circulation 114:82-96

43. Jenkins DJ, Wolever TM, Taylor RH et al (1981) Glycemic index of foods: a physiological basis for carbohydrate exchange. Am J Clin Nutr 34:362-366

44. Foster-Powell K, Holt SH, Brand-Miller JC (2002) International table of glycemic index and glycemic load values: 2002. Am J Clin Nutr 76:5-56

45. Anderson JW, Story L, Sieling B, Chen WJ, Petro MS, Story J (1984) Hypocholesterolemic effects of oat-bran or bean intake for hypercholesterolemic men. Am J Clin Nutr 40:1146-1155

46. Wursch P, Acheson K, Koellreutter B, Jequier E (1988) Metabolic effects of instant bean and potato over 6 hours. Am J Clin Nutr 48:1418-1423

47. Cobiac L, McArthur R, Nestel PJ (1990) Can eating baked beans lower plasma cholesterol? Eur J Clin Nutr 44:819-822

48. Fleming SE, Shaheen SM (1988) Repeated consumption of highfiber breakfasts: effects on postprandial glucose and insulin responses after breakfast and lunch. Am J Clin Nutr 47:859-867

49. Fruhbeck G, Monreal I, Santidrian S (1997) Hormonal implications of the hypocholesterolemic effect of intake of field beans (Vicia faba L.) by young men with hypercholesterolemia. Am J Clin Nutr 66:1452-1460

50. Jang Y, Lee JH, Kim OY, Park HY, Lee SY (2001) Consumption of whole grain and legume powder reduces insulin demand, lipid peroxidation, and plasma homocysteine concentrations in patients 
with coronary artery disease: randomized controlled clinical trial. Arterioscler Thromb Vasc Biol 21:2065-2071

51. Nestel P, Cehun M, Chronopoulos A (2004) Effects of long-term consumption and single meals of chickpeas on plasma glucose, insulin, and triacylglycerol concentrations. Am J Clin Nutr 79:390-395

52. Winham DM, Hutchins AM (2007) Baked bean consumption reduces serum cholesterol in hypercholesterolemic adults. Nutr Res 27:380-386

53. Winham DM, Hutchins AM, Johnston CS (2007) Pinto bean consumption reduces biomarkers for heart disease risk. J Am Coll Nutr 26:243-249

54. Pittaway JK, Ahuja KD, Robertson IK, Ball MJ (2007) Effects of a controlled diet supplemented with chickpeas on serum lipids, glucose tolerance, satiety and bowel function. J Am Coll Nutr 26:334-340

55. Simpson HC, Simpson RW, Lousley S et al (1981) A high carbohydrate leguminous fibre diet improves all aspects of diabetic control. Lancet 1:1-5

56. Kinmonth AL, Angus RM, Jenkins PA, Smith MA, Baum JD (1982) Whole foods and increased dietary fibre improve blood glucose control in diabetic children. Arch Dis Child 57:187-194

57. Riccardi G, Rivellese A, Pacioni D, Genovese S, Mastranzo P, Mancini M (1984) Separate influence of dietary carbohydrate and fibre on the metabolic control in diabetes. Diabetologia 26:116-121

58. Karlstrom B, Vessby B, Asp NG, Boberg M, Lithell H, Berne C (1987) Effects of leguminous seeds in a mixed diet in non-insulindependent diabetic patients. Diabetes Res 5:199-205

59. O'Dea K, Traianedes K, Ireland P et al (1989) The effects of diet differing in fat, carbohydrate, and fiber on carbohydrate and lipid metabolism in type II diabetes. J Am Diet Assoc 89:1076-1086

60. Anderson JW, Zeigler JA, Deakins DA et al (1991) Metabolic effects of high-carbohydrate, high-fiber diets for insulindependent diabetic individuals. Am J Clin Nutr 54:936-943

61. Singh RB, Rastogi V, Rastogi SS, Niaz MA, Beegom R (1996) Effect of diet and moderate exercise on central obesity and associated disturbances, myocardial infarction and mortality in patients with and without coronary artery disease. J Am Coll Nutr 15:592-601

62. Chandalia M, Garg A, Lutjohann D, von Bergmann K, Grundy SM, Brinkley LJ (2000) Beneficial effects of high dietary fiber intake in patients with type 2 diabetes mellitus. N Engl J Med 342:1392-1398
63. Giacco R, Parillo M, Rivellese AA et al (2000) Long-term dietary treatment with increased amounts of fiber-rich low-glycemic index natural foods improves blood glucose control and reduces the number of hypoglycemic events in type 1 diabetic patients. Diabetes Care 23:1461-1466

64. Hall RS, Johnson SK, Baxter AL, Ball MJ (2005) Lupin kernel fibre-enriched foods beneficially modify serum lipids in men. Eur J Clin Nutr 59:325-333

65. Van de Laar FA, Lucassen PL, Akkermans RP, Van de Lisdonk EH, Rutten GE, Van Weel C (2005) Alpha-glucosidase inhibitors for type 2 diabetes mellitus. Cochrane Database Syst Rev Issue 2, Art. no.: CD003639. doi:10.1002/14651858.CD003639. pub2

66. US Department of Health and Human Services, Food and Drug Administration Center for Drug Evaluation and Research (2008) Guidance for industry diabetes mellitus: developing drugs and therapeutic biologics for treatment and prevention (DRAFT GUIDANCE). Available from http://www.fda.gov/cder/guidance/ 7630dft.pdf, accessed 24 April, 2009

67. Jenkins DJ, Wolever TM, Taylor RH et al (1980) Rate of digestion of foods and postprandial glycaemia in normal and diabetic subjects. BMJ 281:14-17

68. Jenkins DJ, Wolever TM, Taylor RH et al (1982) Slow release dietary carbohydrate improves second meal tolerance. Am J Clin Nutr 35:1339-1346

69. Matsuda M, DeFronzo RA (1999) Insulin sensitivity indices obtained from oral glucose tolerance testing: comparison with the euglycemic insulin clamp. Diabetes Care 22:1462-1470

70. Sievenpiper JL, Jenkins DJA, Josse RG, Leiter AL, Vuksan V (2000) Body composition does not affect the intra-subject coefficient of variation of $75 \mathrm{~g}$ oral glucose tolerance test (OGTT) outcome but might influence the reproducibility of diagnosis. Can J Diabetes Care 24:39-46

71. Sievenpiper JL, Kendall CWC, Bazinet RP, Wong JM, Esfahani A, Jenkins DJA (2008) Pulses as part of high-fibre interventions for glycemic control: a meta-analysis of controlled trials. J Clin Lipidol 2:S139

72. Sievenpiper JL, Esfahani A, Wong JM et al (2009) Effect of nonoil seed pulses on glycemic control: a meta-analysis of randomized controlled experimental trials in humans. FASEB J 23:213217 (Abstract) 